Check for updates

Cite this: RSC Adv., 2019, 9, 32375

Received 29th August 2019

Accepted 24th September 2019

DOI: $10.1039 / c 9 r a 06843 k$

rsc.li/rsc-advances

\title{
Facile synthesis of novel dithioacetal-naphthalene derivatives as potential activators for plant resistance induction $\uparrow$
}

\begin{abstract}
R. J. Ji, $\neq^{a}$ W. M. Shi,,$^{a}$ D. Y. Tian, ${ }^{a}$ G. P. Zhang (D) ${ }^{* a}$ and H. Wang ${ }^{\star b}$
In this paper, a series of novel dithioacetal-naphthalenes were designed and synthesized for plant immunity. Their antiviral activities were evaluated against tobacco mosaic virus (TMV) and cucumber mosaic virus (CMV). The results indicated that most compounds exhibited better activity against CMV than against TMV. These dithioacetal derivatives also displayed good bacterial activity against rice bacterial leaf blight. Among them, compound S16 exhibited relatively good anti-CMV, anti-TMV, and antibacterial activity. Structure-activity relationships indicated that introducing the naphthalene moiety enhanced their activities for plant resistance induction. Therefore, the basic motif of compound S16 could be the most promising candidate for further structural optimization to develop a potential activator for plant resistance induction.
\end{abstract}

\section{Introduction}

Plant viruses are pathogenic to plants, infecting many plants, especially vegetables, including pepper, tomato, cucumber, and so on. ${ }^{1}$ Owing to the diversity of virus species, the different transmission mechanisms and the mutability of viruses under field conditions, it is extremely difficult to control viral infection, ${ }^{2}$ which results in massive economic losses each year. ${ }^{3-5}$ Although numerous chemical and biological controls have been applied, there have been few efficient compounds that can protect plants completely from virus infection. ${ }^{6}$ In the field, the reported antiviral agents, ${ }^{7}$ such as ninominomycin, dufulin, ribavirin, lentinan polysaccharide, emodinmethyl ether, morinanidine hydrochloride, chlorbromo-isocyanurate, DHT, DADHT, and chitooligosaccharide, are often effective only against one virus while disabled against the others. Furthermore, the agents with a preventive effect are less than $60 \%$ among the antiviral agents. ${ }^{8}$ Therefore the development of new antiviral agents with an efficient broad-spectrum is extremely urgent.

In fact, plants have self-defend system and can resist the infection of bacteria, mold and viruses. ${ }^{9}$ Such self-defensive ability is commonly induced by some external or internal

${ }^{a}$ Chemistry and Material Science College, Huaibei Normal University, Huaibei 235000, China.E-mail: hbzgp-1@163.com

${ }^{b}$ Institute for Plant Protection and Soil Science, Hubei Academy of Agricultural Sciences, Nanhu Road 6, Wuhan 430064, China. E-mail: wanghua4@163.com

$\dagger$ Electronic supplementary information (ESI) available. See DOI: 10.1039/c9ra06843k

\$ These authors contributed to this work equally. elicitors. Based on plant immune resistance, these elicitors are developed into antivirus agents and used for the prevention and treatment of plant virus..$^{10-14}$ In the development of the chemical antivirus, people mainly consider the virus itself. Many of these reported drugs suffer from low effectiveness and narrowspectrum antiviral property. In addition, the absolute parasite of the virus on the host is closely related to the plant. In general, two factors of virus inhibition and plant activation must be considered together to develop efficient broad-spectrum antiviral agents.

Dithioacetal and its derivatives have extensive biological activities, such as antibacterial, ${ }^{15}$ antileishmanial, ${ }^{16}$ antiviral, ${ }^{17}$ and antifungal. ${ }^{18,19}$ Song et al. found that dithioacetal derivative 6f (Fig. 1) exerted markedly curative and protective activities against PVY and CMV. ${ }^{20}$ Further research results by this group showed that dithioacetal C14 (Fig. 1) elicited excellent curative and protective activities against PVY, CMV and TMV. ${ }^{21}$ Their mechanism associated with the change of SOD, CAT, and POD was also demonstrated. Based on above, introduction of plant immune elicitors, dithiacetal compounds could be developed into novel antiviral agents with effectiveness and spectrum width.
$6 f$

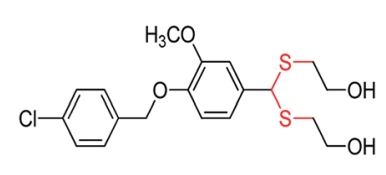

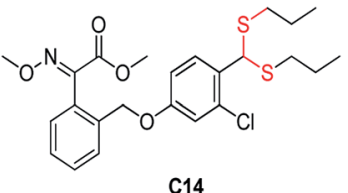

C14
Fig. 1 The structures of the reported dithioacetal compounds with high activity. 
Naproxen acetic acid and naproxen sodium derivatives are important plant regulators for agriculture. ${ }^{22}$ These regulators can promote plant growth and chlorophyll synthesis. Especially it can promote the formation of adventitious roots and roots with fruit expansion and anti-falling function. We then introduced the plant regulated naphthalene group to dithioacetal skeleton (Fig. 2). Moreover, their bioactivities against cucumber mosaic virus and tobacco mosaic virus were subsequently evaluated and preliminary structure-activity relationship was concluded.

\section{Results and discussion}

The condensation reaction of aromatic aldehyde bearing naphthalene ring (M1) with allyl mercaptan was chosen as a model reaction to optimize the reaction conditions and the results are summarized in Table 1 . It was observed that the reaction failed to give the product without the catalyst (Table 1 , entry 1). In initial studies, different catalysts were screened in dichloromethane as the solvent $(1 \mathrm{~mL})$ at $40{ }^{\circ} \mathrm{C}$ (Table 1 , entry 2 and 3). It was found that the acid ionic liquid (BIL) could effectively catalyzed the condensation reaction. The effects of solvents were then evaluated by using ionic liquid (BIL) as the catalyst (Table 1, entry 3-6). Dichloroethane (Table 1, entry 5) was found to be an optimal solvent, giving $\mathbf{S 1}$ in a good yield (up to $97 \%$ ). Almost no change in reactivity was observed when the loading of $\mathrm{BIL}-\mathrm{HSO}_{4}$ was reduced from $10 \mathrm{~mol} \%$ to $5 \mathrm{~mol} \%$ (Table 1, entries 5 and 7). However, the product decreased from $97 \%$ to $69 \%$ when the catalyst load was reduced from $5 \mathrm{~mol} \%$ to $1 \mathrm{~mol} \%$ (Table 1, entries 7 and 8).

The reusability of the catalyst $\left(\mathrm{BIL}-\mathrm{HSO}_{4}\right.$ ) was investigated and the results are described in Fig. 3. The reaction mixture was concentrated under reduced pressure to remove the dichloromethane. $20 \mathrm{~mL}$ water was added to the mixture. Then the catalyst was recovered by using simple filtration technique. The filtrate containing ionic liquid was dried over vacuum to remove excess water. This catalyst was directly subjected to the condensation reaction using the model reaction with our optimized reaction conditions. It is important to note that the recycled catalysts produced excellent yields of dithioacetal (S1)

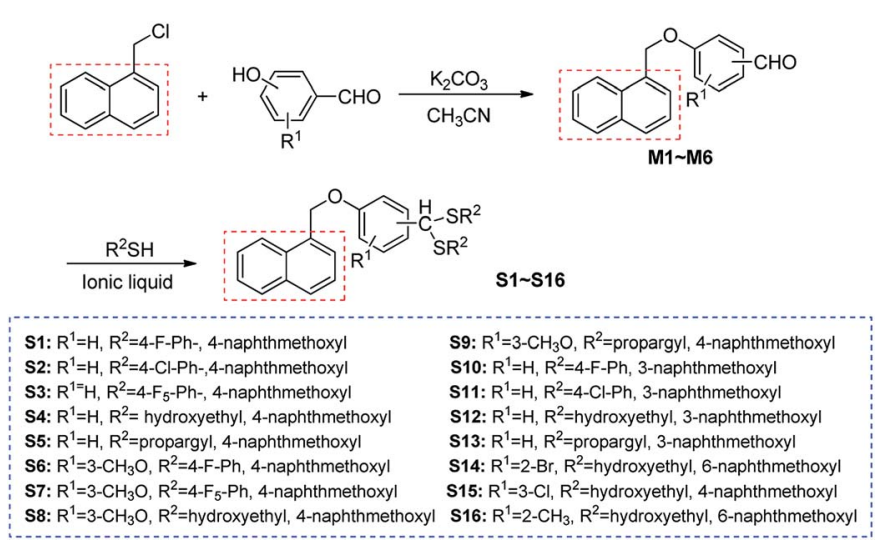

Fig. 2 The synthetic routes of novel dithioacetal derivatives.
Table 1 Optimizations of reaction conditions for the synthesis of S1 catalyzed by ionic liquid (BIL)

\begin{tabular}{|c|c|c|c|c|c|}
\hline Entry $^{a}$ & Catalyst (mol\%) & Solvent & Temp $/{ }^{\circ} \mathrm{C}$ & Time & Yield $^{b} / \%$ \\
\hline 1 & - & DCM & 40 & 6 & 0 \\
\hline 2 & $\mathrm{ZrCl}_{4}(10)$ & DCM & 40 & 6 & 71 \\
\hline 3 & $\mathrm{BIL}-\mathrm{HSO}_{4}(10)$ & DCM & 40 & 6 & 81 \\
\hline 4 & $\mathrm{BIL}^{-\mathrm{HSO}_{4}}(10)$ & $\mathrm{CH}_{3} \mathrm{CN}$ & 80 & 6 & 67 \\
\hline 5 & 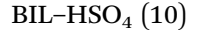 & DCE & 80 & 6 & 97 \\
\hline 6 & $\mathrm{BIL}^{-\mathrm{HSO}_{4}}(10)$ & DOX & 110 & 6 & 87 \\
\hline 7 & BIL- $\mathrm{HSO}_{4}(5)$ & DCE & 80 & 12 & 96 \\
\hline 8 & 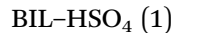 & DCE & 80 & 12 & 69 \\
\hline
\end{tabular}

${ }^{a}$ Reaction conditions: aldehyde $(1.0 \mathrm{mmol})$, thiol $(1.0 \mathrm{mmol})$, solvent $(1.0 \mathrm{~mL}) .{ }^{b}$ Isolated yield.

in $91-94 \%$, respectively (Fig. 3). It was observed that the yields were consistent without significant loss in its catalytic activity.

Under these optimized reaction conditions (Table 1, entry 7), substituted aldehydes bearing naphthalene ring (M1-M6), using acid ionic liquid (BIL), ${ }^{23}$ reacted with different thiols to generate novel dithioacetals S1-S16 with excellent yields of 83$97 \%$.

First, compounds S1-S16 were measured for their phytotoxic activity against tobacco. ${ }^{24}$ The data of phytotoxic activity at 500 $\mu \mathrm{g} \mathrm{mL} \mathrm{m}^{-1}$ indicated that compounds S1-S16 showed no toxicity to the tested plant.

The in vivo anti-TMV activities of target compounds S1-S16 at the concentration of $500 \mu \mathrm{g} \mathrm{mL}^{-1}$ were evaluated through the half leaf method. ${ }^{25-29}$ The results were shown in Table 1. Ningnanmycin and dufulin were used as the controls. Most of the title compounds exhibited good antiviral activities against CMV. Compounds S5, S8, S12 and S16 possessed excellent curative and protective activities from $61.5 \%$ to $71.3 \%$, which was significantly greater than those of the controls with the vicinity of 50\%. Compounds S1, S4, S9 and S13 exhibited good curative and protective activities with $55 \%$ or so, which were slightly exceeded than those of controls. Other compounds maintained moderate antiviral activities. The results of the activity against TMV showed that the target compounds displayed common

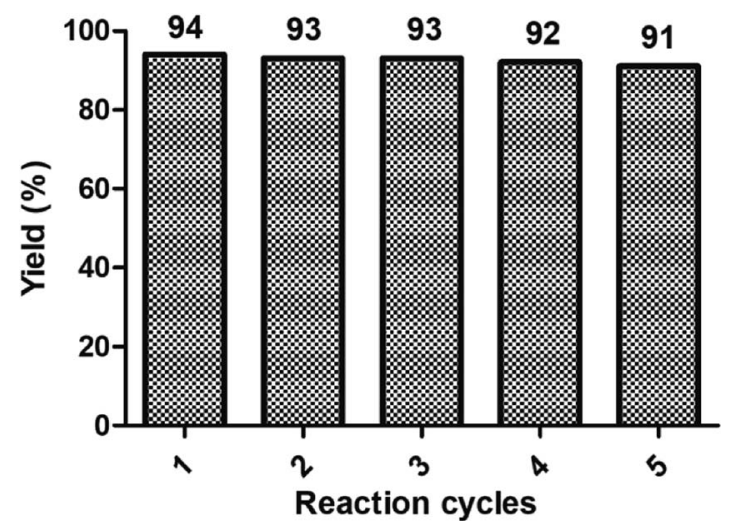

Fig. 3 Reusability of ionic liquid $\left(\mathrm{BIL}-\mathrm{HSO}_{4}\right)$ for the synthesis of compound S1. 
Table 2 Antiviral activity of the title compounds (S1-S16) against TMV and CMV at $500 \mu \mathrm{g} \mathrm{mL}^{-1 a}$

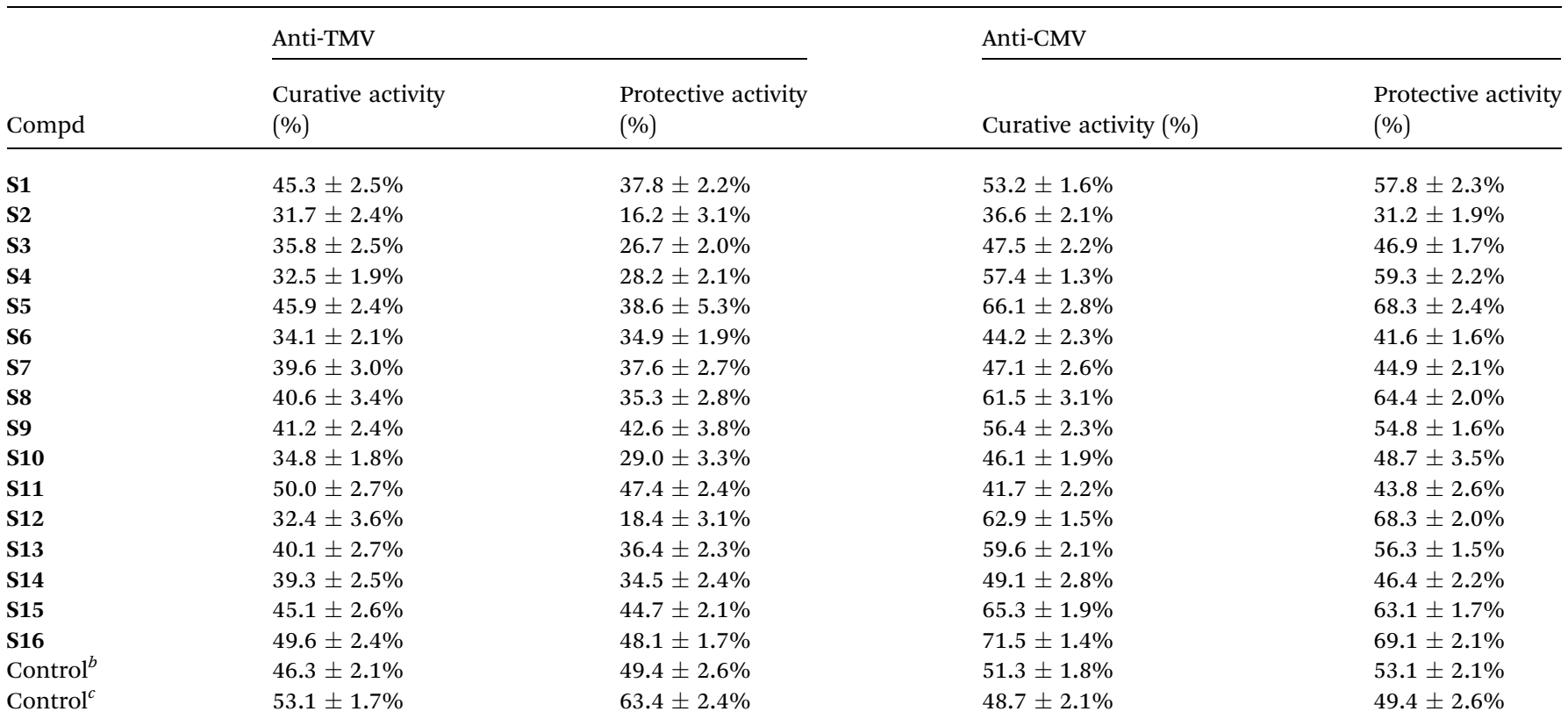

${ }^{a}$ The reaction was conducted in anoxic conditions. ${ }^{b}$ Dufulin was used as the control. ${ }^{c}$ Ningnanmycin was used as the control.

inhibitory effects (Table 2). The curative activities of S1 (45.3\%), S5 (45.9\%), S11 (50.0\%) and S16 (49.6\%) against TMV were similar to those of dufulin (46.3\%), slightly lower than ningnanmycin (53.1\%). The protective activities against TMV of S11 $(47.4 \%)$ and $\mathbf{S 1 6}(49.6 \%)$ were similar to those of dufulin (49.4\%), much less than ningnanmycin (63.4\%). Moreover, the activity of the title compound $\mathbf{S 8}$ is excess than those of the accordingly intermediates referring to the literature. ${ }^{\mathbf{2}}$

Bioassay results indicated that the introduction of naphthalene moiety to the dithioacetals can effectively improve the antiviral activity. In addition, among these compounds S1-S5, the $\mathrm{R}^{2}$ groups affect the antiviral activity $(\mathbf{S 1}, \mathbf{S 5}>\mathbf{S 2}, \mathbf{S 3}, \mathbf{S 4})$, with 1-naphthalene-methyl moiety at the para-position of dithioacetals in anti-TMV activity. The effect of the $\mathrm{R}^{2}$ groups in anti-CMV activity is more obvious (S5 $>$ S4 $>$ S1 $>$ S3 $>$ S2). Compound S5 or S4 exhibited high activities when $\mathrm{R}^{2}$ is hydroxyethyl or propargyl moiety. To study the influence of the $\mathrm{R}^{1}$ group, among these compounds $\mathbf{S 6 - S 9}$, its effect on activity is quite small with the $\mathrm{CH}_{3} \mathrm{O}$ group at the meta-position in antiTMV activity. Nevertheless, compound $\mathbf{s 8}$ with the $\mathrm{CH}_{3} \mathrm{O}$ group at the $\mathrm{R}^{1}$ position and the hydroxyethyl group showed good activity against CMV, further illustrate that the hydroxyethyl group is favorable for activity (S4, S8 and S12) regardless of naphthalene and $\mathrm{R}^{1}$ position on dithioacetals. To screen out high active compounds, the hydroxyethyl group was choosed to change the $\mathrm{R}^{1}$ group and naphthalene position of the title compounds, the compound $\mathbf{S 1 6}$ exhibited best activity against CMV and TMV. The results indicate that $R_{1}$ and naphthalene moiety is important for antiviral activities. Meanwhile, the hydroxyethyl group is indispensable for the antiviral activities of these compounds.
The antibacterial activities of the title compounds (S1-S16) against rice bacterial leaf blight were evaluated by turbidimeter tests. $^{30}$ Bismerthiazol was used as the positive control. ${ }^{31}$ The results showed that the title compounds displayed moderate to good antibacterial activities (Table 3). Among them, compounds S4, S7, S8 exhibited good in vitro antibacterial activity at concentrations of 200 and $100 \mu \mathrm{g} \mathrm{mL}{ }^{-1}$, which were slightly superior to bismerthiazol (82.3 and 57.8\%, respectively). Compounds S2, S6, S15 and S16 displayed moderate antibacterial activity, compared to that of control. Other compounds maintain low antibacterial activities. From the Table 3, SAR is easier to summarize. When $\mathrm{R}^{1}=\mathrm{H}$ and 1-naphthalene-methoxy at the 4-position, the order of activities (S1-S5) from high to low activity at $100 \mu \mathrm{g} \mathrm{mL} \mathrm{m}^{-1}$ was hydroxyethyl (71.1\%), 4-Cl-Ph(57.0\%), 4-F-Ph- (42.4\%), 4- $\mathrm{F}_{5}-\mathrm{Ph}-(33.0 \%)$, propargyl (19.9\%). Different dithioacetal groups obviously affected the activities. When $\mathrm{R}^{1}$ was $3-\mathrm{CH}_{3} \mathrm{O}$ and naphthalene-methoxy at 4-position, the order of activities (S6-S9) at $100 \mu \mathrm{g} \mathrm{mL} \mathrm{mb}^{-1}$ was hydroxyethyl (71.7\%), 4- $\mathrm{F}_{5}-\mathrm{Ph}-(70.6 \%), 4-\mathrm{F}-\mathrm{Ph}-(63.1 \%)$, propargyl (46.8\%). Among them, 3- $\mathrm{CH}_{3} \mathrm{O}$ group could increase the activity. When $\mathrm{R}^{1}$ was $\mathrm{H}$-group and naphthalene-methoxy at 3-position, the order of activities (S10-S13) was hydroxyethyl (51.8\%), 4-F-Ph(30.3\%), 4-Cl-Ph- (25.6\%), propargyl (22.8\%). When $\mathrm{R}^{2}$ was selected for the hydroxyethyl group, the sequence activities of compounds were S15 (63.2\%), S16 (57.1\%), S14 (39.6\%). The above results further suggested that the dithioacetal hydroxyethyl group of the title compounds is critical for the activity and the $\mathrm{R}^{1}$ substituted and 1-naphthalene-methoxy of the derivatives influenced the antibacterial activity.

To investigate the stability property of aromatic dithioacetals, compound $\mathbf{S 1}$ was selected to investigate in different conditions. First, compound $\mathbf{S 1}$ was stable under the condition 
Table 3 Activities of the title compounds (S1-S16) against rice bacterial leaf blight

\begin{tabular}{|c|c|c|c|c|c|}
\hline \multirow[b]{2}{*}{ Compd } & \multicolumn{2}{|c|}{ Inhibition $^{a}(\%)$} & \multirow[b]{2}{*}{ Compd } & \multicolumn{2}{|c|}{ Inhibition $^{a}(\%)$} \\
\hline & $200 \mu \mathrm{g} \mathrm{mL}^{-1}$ & $100 \mu \mathrm{g} \mathrm{mL}^{-1}$ & & $200 \mu \mathrm{g} \mathrm{mL}^{-1}$ & $\begin{array}{l}100 \mu \mathrm{g} \\
\mathrm{mL}^{-1}\end{array}$ \\
\hline S1 & $68.1 \pm 1.8$ & $42.4 \pm 2.2$ & S9 & $66.2 \pm 3.5$ & $46.8 \pm 5.8$ \\
\hline S2 & $98.9 \pm 2.2$ & $57.0 \pm 4.5$ & S10 & $79.2 \pm 2.6$ & $30.3 \pm 5.2$ \\
\hline S3 & $74.5 \pm 3.8$ & $33.0 \pm 2.4$ & S11 & $49.8 \pm 1.3$ & $25.6 \pm 5.4$ \\
\hline S6 & $84.7 \pm 3.0$ & $63.1 \pm 0.8$ & S14 & $69.1 \pm 4.6$ & $39.6 \pm 5.0$ \\
\hline S7 & $93.1 \pm 3.4$ & $70.6 \pm 3.7$ & S15 & $98.9 \pm 3.9$ & $63.2 \pm 4.5$ \\
\hline S8 & $100.0 \pm 1.6$ & $71.7 \pm 3.2$ & S16 & $93.3 \pm 3.4$ & $57.1 \pm 1.1$ \\
\hline Control $^{b}$ & $82.3 \pm 4.3$ & $57.9 \pm 3.1$ & & & \\
\hline
\end{tabular}

of $0.1 \mathrm{M}$ hydrochloric acid solution. Subsequently, compound S1 was also stable in $0.1 \mathrm{M} \mathrm{NaOH}$. The results indicated that aromatic dithioacetals acetals displayed much better stability than acetals.

\section{Conclusions}

In summary, a series of novel dithioacetals-naphthalene, total sixteen compounds have been designed and synthesized with moderate yields. Their structures have been fully confirmed. Bioassay results showed that some compounds exhibited good anti-CMV activities, antibacterial activity and moderate antiTMV activities in vivo. Among them, compound S16 in particularly showed the potent activity against CMV, TMV and good antibacterial activity. Therefore, the basic motif of $\mathbf{S 1 6}$ can be used as lead compound for further development.

\section{Experimental}

\section{General information}

All of the reagents were purchased from commercial suppliers and used without further purification. All of the solvents were used without further purification and drying before use. Thinlayer chromatography with UV detection was conducted on silica gel GF254. The melting points of the products were determined with a WRX-4 microscopic melting point meter (Shanghai Yice Apparatus \& Equipment Co., Ltd., China) with an uncorrected thermometer. ${ }^{1} \mathrm{H}$, and ${ }^{13} \mathrm{C}$ nuclear magnetic resonance (NMR) spectra were recorded on a Bruker Ascend-400 spectrometer (Bruker, Germany) in $\mathrm{CDCl}_{3}$ solution with tetramethylsilane as internal standard. High resolution mass spectral (HRMS) data were determined with Thermo Scientific Q Exactive (Thermo).

Preparation of aromatic aldehydes 1. Chloromethyl naphthalene (I, $10 \mathrm{mmol}$ ) was added to a vial containing acetonitrile (40 $\mathrm{mL}$ ), and hydroxyl substituted benzaldehyde (II, $10 \mathrm{mmol}$ ) and potassium carbonate $(10 \mathrm{mmol})$ were then added. After the mixture was stirred and refluxed for $6 \mathrm{~h}$, the solvent was removed in vacuo. The residue was purified by column chromatography on silica gel (EtOAc/hexane as eluent $=1: 20$ ) affording the aromatic aldehydes (M1-M6).

4-(Naphthalen-1-ylmethoxy)benzaldehyde (M1). White solid, mp 122-123 ${ }^{\circ} \mathrm{C}$, yield 95\%; ${ }^{1} \mathrm{H}$ NMR (400 MHz, $\left.\mathrm{CDCl}_{3}\right) \delta 9.91(\mathrm{~s}$, 1H), 8.07-7.99 (m, 1H), 7.96-7.83 (m, 4H), 7.65-7.43 (m, 4H), $7.16(\mathrm{~d}, J=8.7 \mathrm{~Hz}, 2 \mathrm{H}), 5.59(\mathrm{~s}, 2 \mathrm{H}) ;{ }^{13} \mathrm{C} \mathrm{NMR}\left(101 \mathrm{MHz}, \mathrm{CDCl}_{3}\right)$ $\delta 190.77$ (s), 163.80 (s), 133.83 (s), 132.04 (s), 131.40 (s), 131.27 (s), 130.27 (s), 129.42 (s), 128.84 (s), 126.77 (s), 126.68 (s), 126.09 (s), 125.30 (s), 123.43 (s), 115.21 (s), 68.95 (s).

3-Methoxy-4-(naphthalen-1-ylmethoxy)benzaldehyde (M2). White solid, mp 111-112 ${ }^{\circ} \mathrm{C}$, yield 94\%; ${ }^{1} \mathrm{H}$ NMR $(400 \mathrm{MHz}$, $\left.\mathrm{CDCl}_{3}\right) \delta 9.84(\mathrm{~s}, 1 \mathrm{H}), 8.04(\mathrm{~d}, J=8.0 \mathrm{~Hz}, 1 \mathrm{H}), 7.92-7.81(\mathrm{~m}, 2 \mathrm{H})$, $7.62-7.48(\mathrm{~m}, 3 \mathrm{H}), 7.48-7.36(\mathrm{~m}, 3 \mathrm{H}), 7.08(\mathrm{~d}, J=8.2 \mathrm{~Hz}, 1 \mathrm{H})$, $5.65(\mathrm{~s}, 2 \mathrm{H}), 3.90(\mathrm{~s}, 3 \mathrm{H}) ;{ }^{13} \mathrm{C} \mathrm{NMR}\left(101 \mathrm{MHz}, \mathrm{CDCl}_{3}\right) \delta 190.91(\mathrm{~s})$, 153.73 (s), $150.30(\mathrm{~s}), 133.79$ (s), 131.28 (s), 131.22 (s), 130.50 (s), 129.14 (s), 128.82 (s), 126.57 (s), 126.54 (s), 126.27 (s), 126.00 (s), 125.35 (s), 123.31 (s), 112.74 (s), 109.60 (s), 69.51 (s), 56.07 (s).

3-(Naphthalen-1-ylmethoxy)benzaldehyde (M3). White solid, mp 95-96 ${ }^{\circ} \mathrm{C}$, yield 97\%; ${ }^{1} \mathrm{H}$ NMR (400 MHz, $\left.\mathrm{CDCl}_{3}\right) \delta 9.99(\mathrm{~s}$, $1 \mathrm{H})$, 8.09-8.00 (m, 1H), 7.94-7.83 (m, 2H), 7.62-7.44 (m, 7H), 7.32-7.26 (m, 1H), 5.54 (s, 2H); ${ }^{13} \mathrm{C}$ NMR (101 MHz, $\mathrm{CDCl}_{3}$ ) $\delta 192.07$ (s), 159.41 (s), 137.91 (s), 133.83 (s), 131.67 (s), 131.51 (s), 130.20 (s), 129.30 (s), 128.78 (s), 126.78 (s), 126.60 (s), 126.03 (s), 125.32 (s), 123.82 (s), 123.60 (s), 122.30 (s), 113.32 (s), 68.94 (s).

3-Chloro-4-(naphthalen-1-ylmethoxy)benzaldehyde (M4). Sandy solid, mp 119-121 ${ }^{\circ} \mathrm{C}$, yield 96\%; ${ }^{1} \mathrm{H}$ NMR (400 MHz, $\mathrm{CDCl}_{3}$ ) $\delta 9.86(\mathrm{~s}, 1 \mathrm{H}), 8.07(\mathrm{~d}, J=8.1 \mathrm{~Hz}, 1 \mathrm{H}), 8.00-7.83(\mathrm{~m}, 3 \mathrm{H}), 7.77$ $(\mathrm{dd}, J=8.5,2.0 \mathrm{~Hz}, 1 \mathrm{H}), 7.66(\mathrm{~d}, J=6.8 \mathrm{~Hz}, 1 \mathrm{H}), 7.63-7.53(\mathrm{~m}$, 2H), 7.53-7.46 (m, 1H), $7.23(\mathrm{~d}, J=8.5 \mathrm{~Hz}, 1 \mathrm{H}), 5.70(\mathrm{~s}, 2 \mathrm{H}) .{ }^{13} \mathrm{C}$ NMR (101 MHz, $\mathrm{CDCl}_{3}$ ) $\delta 189.67$ (s), 159.00 (s), 133.77 (s), 131.45 (s), 131.10 (s), 130.70 (s), 130.55 (s), 130.29 (s), 129.36 (s), 128.85 (s), 126.67 (s), 126.22 (s), 126.11 (s), 125.28 (s), 124.47 (s), 123.22 (s), 113.32 (s), 69.73 (s).

2-Bromo-5-(naphthalen-1-ylmethoxy)benzaldehyde (M5). Red solid, mp 129-131 ${ }^{\circ} \mathrm{C}$, yield 94\%; ${ }^{1} \mathrm{H}$ NMR (400 MHz, $\mathrm{CDCl}_{3}$ ) $\delta 10.35(\mathrm{~s}, 1 \mathrm{H}), 8.06-7.99(\mathrm{~m}, 1 \mathrm{H}), 7.95-7.86(\mathrm{~m}, 2 \mathrm{H}), 7.65(\mathrm{~d}, J=$ $3.2 \mathrm{~Hz}, 1 \mathrm{H}), 7.63-7.52(\mathrm{~m}, 4 \mathrm{H}), 7.49(\mathrm{dd}, J=8.1,7.2 \mathrm{~Hz}, 1 \mathrm{H}), 7.15$ $(\mathrm{dd}, J=8.8,3.2 \mathrm{~Hz}, 1 \mathrm{H}), 5.54(\mathrm{~s}, 2 \mathrm{H}) .{ }^{13} \mathrm{C} \mathrm{NMR}\left(101 \mathrm{MHz}, \mathrm{CDCl}_{3}\right)$ 
$\delta 191.68$ (s), 158.46 (s), 134.72 (s), 134.09 (s), 133.82 (s), 131.46 (s), 131.29 (s), 129.41 (s), 128.79 (s), 126.86 (s), 126.64 (s), 126.05 (s), 125.26 (s), 123.83 (s), 123.50 (s), 118.30 (s), 113.94 (s), 69.20 (s).

5-Methyl-2-(naphthalen-1-ylmethoxy)benzaldehyde (M6). White solid, mp 114-116 ${ }^{\circ} \mathrm{C}$, yield 93\%; ${ }^{1} \mathrm{H}$ NMR (400 MHz, $\mathrm{CDCl}_{3}$ ) $\delta 10.43(\mathrm{~s}, 1 \mathrm{H}), 8.06-8.02(\mathrm{~m}, 1 \mathrm{H}), 7.96-7.82(\mathrm{~m}, 2 \mathrm{H}), 7.67$ (d, $J=$ $2.1 \mathrm{~Hz}, 1 \mathrm{H}), 7.63-7.42(\mathrm{~m}, 4 \mathrm{H}), 7.38$ (dd, $J=8.5,2.3 \mathrm{~Hz}, 1 \mathrm{H}), 7.10$ $(\mathrm{d}, J=8.5 \mathrm{~Hz}, 1 \mathrm{H}), 5.60(\mathrm{~s}, 2 \mathrm{H}), 2.33(\mathrm{~s}, 3 \mathrm{H}) ;{ }^{13} \mathrm{C} \mathrm{NMR}(101 \mathrm{MHz}$, $\left.\mathrm{CDCl}_{3}\right) \delta 189.88(\mathrm{~s}), 159.26$ (s), 136.52 (s), 133.81 (s), 131.60 (s), 131.40 (s), 130.62 (s), 129.30 (s), 128.84 (s), 128.47 (s), 126.67 (s), 126.53 (s), 126.06 (s), 125.26 (s), 125.12 (s), 123.36 (s), 113.24 (s), $69.38(\mathrm{~s}), 20.30(\mathrm{~s})$.

Procedure for synthesis of dithioacetal derivatives (S1-S16). Aromatic aldehyde (M, $1.0 \mathrm{mmol}$ ), thiols (1.0 mmol), $2.5 \mathrm{~mol} \%$ Brønsted acidic ionic liquid were added to $1.0 \mathrm{~mL}$ of dichloromethane. The mixture was stirring at $40{ }^{\circ} \mathrm{C}$ for $3 \mathrm{~h}$. The resulting mixture was concentrated under reduced pressure to give crude product. $5 \mathrm{~mL}$ water was added to the crude product and continues to stirring for $0.5 \mathrm{~h}$. Then the water with ionic liquid was removed. Finally, the crude product was purified by column chromatography using hexane/EtOAc $(1: 2$, v/v) or recrystallization with alcohol.

Bis(4-fluorophenyl)-4-(naphthalen-1-ylmethoxy)-phenyldithioacetal (S1). White solid, mp 103-105 ${ }^{\circ} \mathrm{C}$, yield $96 \% ;{ }^{1} \mathrm{H}$ NMR (400 MHz, $\left.\mathrm{CDCl}_{3}\right) \delta 8.05(\mathrm{~d}, J=7.4 \mathrm{~Hz}, 1 \mathrm{H}), 7.95-7.86(\mathrm{~m}$, $2 \mathrm{H}), 7.61-7.47(\mathrm{~m}, 4 \mathrm{H}), 7.37-7.29(\mathrm{~m}, 4 \mathrm{H}), 7.24(\mathrm{~d}, J=8.6 \mathrm{~Hz}$, 2H), 7.01-6.91 (m, 6H), 5.49 (s, 2H), 5.26 (s, 1H); ${ }^{13} \mathrm{C}$ NMR (101 $\mathrm{MHz} \mathrm{CDCl}_{3}$ ) $\delta 164.08(\mathrm{~s}), 161.61(\mathrm{~s}), 158.62(\mathrm{~s}), 135.71(\mathrm{~d}, J=8.4$ Hz), 133.80 (s), 132.05 (s), 131.72 (s), 131.49 (s), 129.30 (s), 129.11 (s), 128.75 (s), 126.57 (d, J=12.3 Hz), 125.97 (s), 125.30 (s), 123.64 (s), 116.07 (s), 115.85 (s), 114.87 (s), 68.72 (s), 61.55 (s); IR (KBr, cm $\left.{ }^{-1}\right) \nu 1604.8(\mathrm{~s}), 1584.1$ (s), 1509.1 (s), 1487.6 (s), 1244.6 (s), 1222.7 (s); HRMS (ES) $m / z$ for $\mathrm{C}_{30} \mathrm{H}_{22} \mathrm{~F}_{2} \mathrm{OS}_{2}[\mathrm{M}+\mathrm{Na}]^{+}$ cacld 523.0972, found 523.0978.

Bis(4-chlorophenyl)-4-(naphthalen-1-ylmethoxy)-phenyl-

dithioacetal (S2). White solid, mp 113-114 ${ }^{\circ} \mathrm{C}$, yield $92 \% ;{ }^{1} \mathrm{H}$ NMR (400 MHz, $\left.\mathrm{CDCl}_{3}\right) \delta$ 8.09-8.02 (m, 1H), 7.96-7.85 (m, 2H), $7.62-7.47(\mathrm{~m}, 4 \mathrm{H}), 7.34-7.20(\mathrm{~m}, 10 \mathrm{H}), 6.98(\mathrm{~d}, J=8.7 \mathrm{~Hz}, 2 \mathrm{H})$, $5.49(\mathrm{~s}, 2 \mathrm{H}), 5.36(\mathrm{~s}, 1 \mathrm{H}) ;{ }^{13} \mathrm{C} \mathrm{NMR}\left(101 \mathrm{MHz}, \mathrm{CDCl}_{3}\right) \delta 158.75(\mathrm{~s})$, 134.21 (s), 134.07 (s), 133.80 (s), 132.72 (s), 132.02 (s), 131.50 (s), 131.28 (s), 129.15 (s), 129.04 (s), 128.75 (s), 126.66 (s), 126.52 (s), 125.98 (s), 125.31 (s), 123.64 (s), 114.99 (s), 68.75 (s), 60.21 (s); IR $\left(\mathrm{KBr}, \mathrm{cm}^{-1}\right) \nu 1505.4(\mathrm{~s}), 1471.6(\mathrm{~s}), 1233.5(\mathrm{~s}), 1091.4(\mathrm{~s}), 1005.4$ (s); HRMS (ES) $m / z$ for $\mathrm{C}_{30} \mathrm{H}_{22} \mathrm{Cl}_{2} \mathrm{OS}_{2}[\mathrm{M}+\mathrm{Na}]^{+}$cacld 555.0381, found 555.0384 .

Bis(pentafluorophenyl)-4-(naphthalen-1-ylmethoxy)-phenyl-

dithioacetal (S3). White solid, mp 137-139 ${ }^{\circ} \mathrm{C}$, yield $83 \% ;{ }^{1} \mathrm{H}$ NMR (400 MHz, $\left.\mathrm{CDCl}_{3}\right) \delta$ 8.05-7.98 (m, 1H), 7.95-7.83 (m, 2H), 7.62-7.51 (m, 3H), 7.50-7.44 (m, 1H), $7.41(\mathrm{~d}, J=8.7 \mathrm{~Hz}, 2 \mathrm{H})$, $6.98(\mathrm{~d}, J=8.7 \mathrm{~Hz}, 2 \mathrm{H}), 5.66(\mathrm{~s}, 1 \mathrm{H}), 5.48(\mathrm{~s}, 2 \mathrm{H}) ;{ }^{13} \mathrm{C} \mathrm{NMR}(101$ $\mathrm{MHz} \mathrm{CDCl}_{3}$ ) $\delta 159.67(\mathrm{~s}), 133.79(\mathrm{~s}), 131.73(\mathrm{~s}), 131.43(\mathrm{~s}), 129.20$ (s), 128.76 (s), 129.11 (s), 128.46 (s), 126.64 (s), 126.55 (s), 125.99 (s), 125.27 (s), 123.52 (s), 115.24 (s), 68.76 (s), 57.63 (s); IR $\left(\mathrm{KBr}, \mathrm{cm}^{-1}\right) \nu 1513.8(\mathrm{~s}), 1487.9$ (s), 1230.5 (s), 1098.2 (s); HRMS (ES) $m / z$ for $\mathrm{C}_{30} \mathrm{H}_{14} \mathrm{~F}_{10} \mathrm{OS}_{2}[\mathrm{M}+\mathrm{Na}]^{+}$cacld 667.0219 , found 667.0216 .
Bis(2-hydroxyethyl)-4-(naphthalen-1-ylmethoxy)-phenyl-

dithioacetal (S4). White solid, mp 105-107 ${ }^{\circ} \mathrm{C}$, yield $94 \% ;{ }^{1} \mathrm{H}$ NMR (400 MHz, $\left.\mathrm{CDCl}_{3}\right) \delta 8.08-8.01(\mathrm{~m}, 1 \mathrm{H}), 7.93-7.84(\mathrm{~m}, 2 \mathrm{H})$, 7.61-7.45 (m, 4H), 7.44-7.37 (m, 2H), 7.07-6.99 (m, 2H), 5.49 (s, $2 \mathrm{H}), 5.07(\mathrm{~s}, 1 \mathrm{H}), 3.74(\mathrm{t}, J=5.5 \mathrm{~Hz}, 4 \mathrm{H}), 2.86(\mathrm{dt}, J=14.0$, $5.8 \mathrm{~Hz}, 2 \mathrm{H}), 2.73(\mathrm{dt}, J=14.0,5.9 \mathrm{~Hz}, 2 \mathrm{H}), 2.17(\mathrm{~s}, 2 \mathrm{H}) ;{ }^{13} \mathrm{C} \mathrm{NMR}$ $\left(101 \mathrm{MHz}, \mathrm{CDCl}_{3}\right.$ ) $\delta 158.77$ (s), 133.81 (s), 132.34 (s), 132.07 (s), 131.52 (s), 129.12 (s), 128.94 (s), 128.72 (s), 126.65 (s), 126.50 (s), 125.95 (s), 125.30 (s), 123.65 (s), 115.13 (s), 68.80 (s), 61.31 (s), 52.68 (s), $35.66(\mathrm{~s}) ; \mathrm{IR}\left(\mathrm{KBr}, \mathrm{cm}^{-1}\right) \nu 3477.8(\mathrm{~s}), 3414.4(\mathrm{~s}), 1617.8$ (s), 1510.5 (s), $1238.2(\mathrm{~s})$; HRMS (ES) $\mathrm{m} / z$ for $\mathrm{C}_{22} \mathrm{H}_{24} \mathrm{O}_{3} \mathrm{~S}_{2}[\mathrm{M}+$ $\mathrm{Na}]^{+}$cacld 423.1059, found 423.1064.

Bis(propenyl)-4-(naphthalen-1-ylmethoxy)-phenyl-dithioacetal (S5). White solid, mp 114-116 ${ }^{\circ} \mathrm{C}$, yield 97\%; ${ }^{1} \mathrm{H} \mathrm{NMR}(400 \mathrm{MHz}$, $\left.\mathrm{CDCl}_{3}\right) \delta$ 8.07-8.01 (m, 1H), 7.92-7.83 (m, 2H), 7.59 (d, $J=$ $6.8 \mathrm{~Hz}, 1 \mathrm{H}), 7.57-7.49(\mathrm{~m}, 2 \mathrm{H}), 7.49-7.43(\mathrm{~m}, 1 \mathrm{H}), 7.38(\mathrm{t}, J=$ $5.8 \mathrm{~Hz}, 2 \mathrm{H}), 7.01(\mathrm{~d}, J=8.7 \mathrm{~Hz}, 2 \mathrm{H}), 5.80$ (ddt, $J=17.1,10.0$, $7.2 \mathrm{~Hz}, 2 \mathrm{H}), 5.48(\mathrm{~s}, 2 \mathrm{H}), 5.18-5.06(\mathrm{~m}, 4 \mathrm{H}), 4.77(\mathrm{~s}, 1 \mathrm{H}), 3.27$ (dd, $J=13.7,7.2 \mathrm{~Hz}, 2 \mathrm{H}), 3.06(\mathrm{dd}, J=13.7,7.2 \mathrm{~Hz}, 2 \mathrm{H}) ;{ }^{13} \mathrm{C}$ NMR (101 MHz, $\mathrm{CDCl}_{3}$ ) $\delta 158.54$ (s), 133.89 (s), 133.82 (s), 132.33 (s), 132.20 (s), 131.55 (s), 129.30 (s), 129.08 (s), 128.72 (s), 126.65 (s), 126.48 (s), 125.94 (s), 125.32 (s), 123.70 (s), 117.52 (s), 114.95 (s), 68.77 (s), 49.99 (s), 35.28 (s); IR (KBr, cm $\left.{ }^{-1}\right) \nu 3351.2(\mathrm{~s})$, 2123.6 (s), 1537.4 (s), 1432.1 (s), 1218.6 (s); HRMS (ES) $m / z$ for $\mathrm{C}_{24} \mathrm{H}_{24} \mathrm{OS}_{2}[\mathrm{M}+\mathrm{Na}]^{+}$cacld 437.1216, found 437.1219.

Bis(4-fluorophenyl)-4-(naphthalen-1-yl-methoxy)-3-

methoxylphenyldithioacetal (S6). White solid, mp 117-119 ${ }^{\circ} \mathrm{C}$, yield 92\%; ${ }^{1} \mathrm{H}$ NMR (400 MHz, $\left.\mathrm{CDCl}_{3}\right) \delta 8.09(\mathrm{~d}, J=8.0 \mathrm{~Hz}, 1 \mathrm{H})$, 7.92-7.79 (m, 2H), 7.60-7.40 (m, 4H), 7.36-7.27 (m, 4H), 6.99$6.89(\mathrm{~m}, 4 \mathrm{H}), 6.87(\mathrm{~d}, J=2.0 \mathrm{~Hz}, 1 \mathrm{H}), 6.81(\mathrm{~d}, J=8.3 \mathrm{~Hz}, 1 \mathrm{H})$, $6.68(\mathrm{dd}, J=8.2,2.1 \mathrm{~Hz}, 1 \mathrm{H}), 5.55$ (s, 2H), 5.19 (s, 1H), 3.80 (s, $3 \mathrm{H}) ;{ }^{13} \mathrm{C}$ NMR (101 MHz, $\mathrm{CDCl}_{3}$ ) $\delta 164.12(\mathrm{~s}), 161.65$ (s), 149.92 (s), 148.06 (s), 135.80 (d, $J=8.5 \mathrm{~Hz}), 133.75$ (s), 132.54 (s), 132.21 (s), 131.37 (s), 129.16 (d, $J=3.4 \mathrm{~Hz}), 128.77$ (d, $J=13.2 \mathrm{~Hz}$ ), 126.31 (d, $J=13.6 \mathrm{~Hz}), 125.86$ (s), 125.30 (s), 123.57 (s), 120.29 (s), 116.06 (s), 115.84 (s), 114.15 (s), 111.46 (s), 69.76 (s), 61.82 (s), 56.02 (s); IR (KBr, cm $\left.{ }^{-1}\right) \nu 1588.4(\mathrm{~s}), 1510.1$ (s), 1486.9 (s), 1466.3 (s), 1265.5 (s), 1227.3 (s), 1136.0 (s); HRMS (ES) $m / z$ for $\mathrm{C}_{31} \mathrm{H}_{24} \mathrm{~F}_{2} \mathrm{O}_{2} \mathrm{~S}_{2}[\mathrm{M}+\mathrm{H}]^{+}$cacld 531.1259, found 531.1257.

Bis(pentafluorophenyl)-4-(naphthalen-1-ylmethoxy)-3-

methoxylphenyldithioacetal (S7). White solid, mp 118-120 ${ }^{\circ} \mathrm{C}$, yield $85 \%$; ${ }^{1} \mathrm{H}$ NMR $\left(400 \mathrm{MHz}, \mathrm{CDCl}_{3}\right) \delta 8.05(\mathrm{~d}, J=7.9 \mathrm{~Hz}, 1 \mathrm{H})$, 7.91-7.82 (m, 2H), 7.58-7.50 (m, 3H), 7.46-7.42 (m, 1H), $7.11(\mathrm{~s}$, $1 \mathrm{H}), 6.83(\mathrm{~s}, 2 \mathrm{H}), 5.64(\mathrm{~s}, 1 \mathrm{H}), 5.54(\mathrm{~s}, 2 \mathrm{H}), 3.88(\mathrm{~s}, 3 \mathrm{H}) .{ }^{13} \mathrm{C} \mathrm{NMR}$ $\left(101 \mathrm{MHz}, \mathrm{CDCl}_{3}\right) \delta 150.23(\mathrm{~s}), 149.20(\mathrm{~s}), 133.74(\mathrm{~s}), 131.84(\mathrm{~s})$, 131.29 (s), 129.06 (s), 128.92 (s), 128.72 (s), 126.42 (s), 126.21 (s), 125.89 (s), 125.27 (s), 123.43 (s), 120.47 (s), 113.97 (s), 110.90 (s), 69.66 (s), 57.91 (s), 56.10 (s); IR (KBr, cm $\left.{ }^{-1}\right) \nu 1514.3$ (s), 1489.8 (s), 1236.1 (s), 1088.2 (s); HRMS (ES) $m / z$ for $\mathrm{C}_{31} \mathrm{H}_{16} \mathrm{~F}_{10} \mathrm{O}_{2} \mathrm{~S}_{2}[\mathrm{M}+$ $\mathrm{Na}]^{+}$cacld 697.0324, found 697.0321.

Bis(2-hydroxyethyl)-4-(naphthalen-1-ylmethoxy)-3-

methoxylphenyldithioacetal (S8). White solid, mp 113-114 ${ }^{\circ} \mathrm{C}$, yield 93\%; ${ }^{1} \mathrm{H}$ NMR $\left(400 \mathrm{MHz}, \mathrm{CDCl}_{3}\right) \delta 8.10(\mathrm{~d}, J=8.1 \mathrm{~Hz}, 1 \mathrm{H})$, $7.91-7.87$ (m, 1H), 7.83 (d, $J=8.3 \mathrm{~Hz}, 1 \mathrm{H}), 7.59$ (d, $J=6.8 \mathrm{~Hz}$, $1 \mathrm{H}), 7.53(\mathrm{td}, J=7.7,1.4 \mathrm{~Hz}, 2 \mathrm{H}), 7.47-7.42(\mathrm{~m}, 1 \mathrm{H}), 7.07(\mathrm{~s}, 1 \mathrm{H})$, $6.91(\mathrm{~s}, 2 \mathrm{H}), 5.56(\mathrm{~s}, 2 \mathrm{H}), 5.03(\mathrm{~s}, 1 \mathrm{H}), 3.89(\mathrm{~s}, 3 \mathrm{H}), 3.74(\mathrm{t}, J=$ 
$5.8 \mathrm{~Hz}, 4 \mathrm{H}), 2.84(\mathrm{dt}, J=14.0,5.8 \mathrm{~Hz}, 2 \mathrm{H}), 2.72(\mathrm{dt}, J=14.0$, $5.9 \mathrm{~Hz}, 2 \mathrm{H}), 2.18(\mathrm{~s}, 2 \mathrm{H}) ;{ }^{13} \mathrm{C} \mathrm{NMR}\left(101 \mathrm{MHz}, \mathrm{CDCl}_{3}\right) \delta 150.33(\mathrm{~s})$, 148.34 (s), 133.76 (s), 133.18 (s), 132.24 (s), 131.39 (s), 128.83 (s), 128.67 (s), 126.36 (s), 126.28 (s), 125.84 (s), 125.32 (s), 123.58 (s), 120.08 (s), 114.18 (s), 111.34 (s), 69.84 (s), 61.34 (s), 56.15 (s), 53.09 (s), $35.72(\mathrm{~s})$; IR (KBr, $\left.\mathrm{cm}^{-1}\right) \nu 3414.5$ (s), 32.46.4 (s), 1508.7 (s), 1466.4 (s), 1259.1 (s), 1212.0 (s), 1141.1 (s), 1004.7 (s); HRMS (ES) $m / z$ for $\mathrm{C}_{23} \mathrm{H}_{26} \mathrm{O}_{4} \mathrm{~S}_{2}[\mathrm{M}+\mathrm{Na}]^{+}$cacld 453.1165, found 453.1161.

Bis(propenyl)-4-(naphthalen-1-ylmethoxy)-3-methoxylphenyldithioacetal (S9). Yellow solid, mp 90-92 ${ }^{\circ} \mathrm{C}$, yield $95 \% ;{ }^{1} \mathrm{H}$ NMR (400 MHz, $\left.\mathrm{CDCl}_{3}\right) \delta 8.10(\mathrm{~d}, J=8.1 \mathrm{~Hz}, 1 \mathrm{H}), 7.92-7.79(\mathrm{~m}$, $2 \mathrm{H}), 7.63-7.40(\mathrm{~m}, 4 \mathrm{H}), 7.06(\mathrm{~d}, J=1.8 \mathrm{~Hz}, 1 \mathrm{H}), 6.94-6.83(\mathrm{~m}$, 2H), 5.79 (ddt, $J=17.1,10.0,7.2 \mathrm{~Hz}, 2 \mathrm{H}), 5.56$ (s, 2H), 5.17-5.04 $(\mathrm{m}, 4 \mathrm{H}), 4.73(\mathrm{~s}, 1 \mathrm{H}), 3.88(\mathrm{~s}, 3 \mathrm{H}), 3.27(\mathrm{dd}, J=13.7,7.1 \mathrm{~Hz}, 2 \mathrm{H})$, $3.06(\mathrm{dd}, J=13.7,7.2 \mathrm{~Hz}, 2 \mathrm{H}) .{ }^{13} \mathrm{C}$ NMR (101 MHz, $\mathrm{CDCl}_{3}$ ) $\delta 150.17$ (s), 148.06 (s), 133.87 (s), 133.76 (s), 133.18 (s), 132.37 (s), $131.40(\mathrm{~s}), 128.78(\mathrm{~s}), 128.67(\mathrm{~s}), 126.34(\mathrm{~s}), 126.26(\mathrm{~s}), 125.82$ (s), 125.35 (s), 123.62 (s), 120.40 (s), 117.54 (s), 114.15 (s), 111.72 (s), 69.84 (s), 56.09 (s), 50.41 (s), 35.34 (s); IR $\left(\mathrm{KBr}, \mathrm{cm}^{-1}\right) \nu$ 3331.2 (s), 2118.4 (s), 1557.4 (s), 1462.1 (s), 1208.9 (s); HRMS (ES) $m / z$ for $\mathrm{C}_{25} \mathrm{H}_{26} \mathrm{O}_{2} \mathrm{~S}_{2}[\mathrm{M}+\mathrm{Na}]^{+}$cacld 445.1266 , found 445.1269 .

Bis(4-fluorophenyl)-3-(naphthalen-1-ylmethoxy)-phenyl-

dithioacetal (S10). White solid, mp 105-106 ${ }^{\circ} \mathrm{C}$, yield $92 \% ;{ }^{1} \mathrm{H}$ NMR (400 MHz, $\left.\mathrm{CDCl}_{3}\right) \delta 8.03(\mathrm{~d}, J=7.8 \mathrm{~Hz}, 1 \mathrm{H}), 7.94-7.84(\mathrm{~m}$, 2H), 7.61-7.51 (m, 3H), 7.49-7.45 (m, 1H), 7.35-7.28 (m, 4H), $7.18(\mathrm{t}, J=7.9 \mathrm{~Hz}, 1 \mathrm{H}), 7.00-6.86(\mathrm{~m}, 7 \mathrm{H}), 5.43(\mathrm{~s}, 2 \mathrm{H}), 5.21(\mathrm{~s}$, $1 \mathrm{H}) ;{ }^{13} \mathrm{C} \mathrm{NMR}\left(101 \mathrm{MHz}, \mathrm{CDCl}_{3}\right) \delta 164.15$ (s), $161.68(\mathrm{~s}), 158.82$ (s), $140.85(\mathrm{~s}), 135.81(\mathrm{~d}, J=8.2 \mathrm{~Hz}), 133.82(\mathrm{~s}), 132.10(\mathrm{~s}), 131.52$ (s), 129.55 (s), 129.08 (s), 128.71 (s), 126.57 (d, $J=18.1 \mathrm{~Hz}$ ), 125.95 (s), 125.28 (s), 123.66 (s), 120.61 (s), 116.05 (s), 115.84 (s), $115.01(\mathrm{~s}), 114.26(\mathrm{~s}), 68.71(\mathrm{~s}), 62.01(\mathrm{~s}) ; \mathrm{IR}\left(\mathrm{KBr}, \mathrm{cm}^{-1}\right) \nu 1643.2$ (s), 1593.7 (s), 1488.9 (s), 1212.1 (s); HRMS (ES) $\mathrm{m} / \mathrm{z}$ for $\mathrm{C}_{30} \mathrm{H}_{22} \mathrm{~F}_{2} \mathrm{OS}_{2}[\mathrm{M}+\mathrm{Na}]^{+}$cacld 523.0972, found 523.0977.

Bis(4-chlorophenyl)-3-(naphthalen-1-ylmethoxy)-phenyl-

dithioacetal (S11). White solid, mp 119-121 ${ }^{\circ} \mathrm{C}$, yield $91 \% ;{ }^{1} \mathrm{H}$ NMR (400 MHz, $\left.\mathrm{CDCl}_{3}\right) \delta 8.04(\mathrm{~d}, J=8.1 \mathrm{~Hz}, 1 \mathrm{H}), 7.97-7.84(\mathrm{~m}$, 2H), 7.66-7.52 (m, 3H), 7.51-7.44 (m, 1H), 7.36-7.18 (m, 9H), 7.06-7.01 (m, 1H), 7.00-6.90 (m, 2H), $5.45(\mathrm{~s}, 2 \mathrm{H}), 5.32(\mathrm{~s}, 1 \mathrm{H})$; ${ }^{13} \mathrm{C}$ NMR (101 MHz, $\mathrm{CDCl}_{3}$ ) $\delta 158.91(\mathrm{~s}), 140.47$ (s), $134.63(\mathrm{~s})$, 134.22 (s), 133.81 (s), 132.51 (s), 132.05 (s), 131.51 (s), 129.68 (s), 129.08 (s), 129.04 (s), 128.72 (s), 126.66 (s), 126.51 (s), 125.95 (s), 125.29 (s), 123.65 (s), 120.60 (s), 115.19 (s), 114.26 (s), 68.72 (s), 60.76 (s); IR (KBr, cm $\left.{ }^{-1}\right) \nu 1544.6$ (s), 1491.6 (s), 1230.5 (s), 1081.4 (s); HRMS (ES) $m / z$ for $\mathrm{C}_{30} \mathrm{H}_{22} \mathrm{Cl}_{2} \mathrm{OS}_{2}[\mathrm{M}+\mathrm{Na}]^{+}$cacld 555.0381, found 555.0376.

Bis(2-hydroxyethyl)-3-(naphthalen-1-ylmethoxy)-phenyl-

dithioacetal (S12). White solid, mp 101-103 ${ }^{\circ} \mathrm{C}$, yield $94 \% ;{ }^{1} \mathrm{H}$ NMR (400 MHz, $\left.\mathrm{CDCl}_{3}\right) \delta 8.07(\mathrm{~d}, J=7.9 \mathrm{~Hz}, 1 \mathrm{H}), 7.97-7.81(\mathrm{~m}$, $2 \mathrm{H}), 7.67-7.42(\mathrm{~m}, 4 \mathrm{H}), 7.34-7.26(\mathrm{~m}, 1 \mathrm{H}), 7.21-7.14(\mathrm{~m}, 1 \mathrm{H})$, 7.07 (d, $J=7.7 \mathrm{~Hz}, 1 \mathrm{H}), 6.98(\mathrm{dd}, J=8.0,2.1 \mathrm{~Hz}, 1 \mathrm{H}), 5.51$ (s, $2 \mathrm{H}), 5.04(\mathrm{~s}, 1 \mathrm{H}), 3.73(\mathrm{~s}, 4 \mathrm{H}), 2.84(\mathrm{dt}, J=14.0,5.8 \mathrm{~Hz}, 2 \mathrm{H}), 2.72$ $(\mathrm{dt}, J=14.0,5.9 \mathrm{~Hz}, 2 \mathrm{H}), 2.10(\mathrm{~s}, 2 \mathrm{H}) ;{ }^{13} \mathrm{C}$ NMR $(101 \mathrm{MHz}$, $\mathrm{CDCl}_{3}$ ) $\delta 159.11$ (s), 141.65 (s), 133.81 (s), 132.10 (s), 131.54 (s), 129.86 (s), 129.08 (s), 128.70 (s), 126.72 (s), 126.50 (s), 125.95 (s), 125.27 (s), 123.70 (s), 120.40 (s), 114.94 (s), 114.24 (s), 68.76 (s), 61.30 (s), 53.17 (s), 35.70 (s); IR (KBr, cm $\left.{ }^{-1}\right) \nu 3415.1$ (s), 3282.6 (s), 1514.8 (s), 1464.7 (s), 1011.2 (s); HRMS (ES) $\mathrm{m} / \mathrm{z}$ for $\mathrm{C}_{22} \mathrm{H}_{24} \mathrm{O}_{3} \mathrm{~S}_{2}[\mathrm{M}+\mathrm{Na}]^{+}$cacld 423.1059, found 423.1062.

Bis(propenyl)-3-(naphthalen-1-ylmethoxy)-phenyl-dithioacetal (S13). White solid, mp 91-93 ${ }^{\circ} \mathrm{C}$, yield 96\%; ${ }^{1} \mathrm{H}$ NMR $(400 \mathrm{MHz}$, $\left.\mathrm{CDCl}_{3}\right) \delta 8.08(\mathrm{~d}, J=7.8 \mathrm{~Hz}, 1 \mathrm{H}), 7.95-7.81(\mathrm{~m}, 2 \mathrm{H}), 7.65-7.44$ $(\mathrm{m}, 4 \mathrm{H}), 7.32-7.26(\mathrm{~m}, 1 \mathrm{H}), 7.17(\mathrm{~d}, J=1.8 \mathrm{~Hz}, 1 \mathrm{H}), 7.05(\mathrm{~d}, J=$ $7.6 \mathrm{~Hz}, 1 \mathrm{H}), 6.97$ (dd, $J=7.9,2.1 \mathrm{~Hz}, 1 \mathrm{H}), 5.80$ (ddt, $J=17.1$, 10.0, 7.2 Hz, 2H), $5.51(\mathrm{~s}, 2 \mathrm{H}), 5.21-5.00(\mathrm{~m}, 4 \mathrm{H}), 4.76(\mathrm{~s}, 1 \mathrm{H})$, $3.28(\mathrm{dd}, J=13.7,7.2 \mathrm{~Hz}, 2 \mathrm{H}), 3.07(\mathrm{dd}, J=13.7,7.2 \mathrm{~Hz}, 2 \mathrm{H}) ;{ }^{13} \mathrm{C}$ NMR (101 MHz, $\left.\mathrm{CDCl}_{3}\right) \delta 159.05$ (s), $141.61(\mathrm{~s}), 133.81(\mathrm{~s}), 133.78$ (s), 132.21 (s), 131.58 (s), 129.64 (s), 129.07 (s), 128.69 (s), 126.75 (s), 126.47 (s), 125.92 (s), 125.30 (s), 123.78 (s), 120.84 (s), 117.64 (s), $114.62(\mathrm{~s}), 114.50(\mathrm{~s}), 68.71$ (s), 50.48 (s), 35.31 (s); IR $\left(\mathrm{KBr}, \mathrm{cm}^{-1}\right) \nu 3321.2(\mathrm{~s}), 2123.4(\mathrm{~s}), 1551.4(\mathrm{~s}), 1458.1(\mathrm{~s}), 1208.9$ (s); HRMS (ES) $m / z$ for $\mathrm{C}_{24} \mathrm{H}_{24} \mathrm{OS}_{2}[\mathrm{M}+\mathrm{Na}]^{+}$cacld 415.1161, found 415.1166 .

Bis(2-hydroxyethyl)-5-(naphthalen-1-ylmethoxy)-2-bromophenyldithioacetal (S14). Red solid, mp 123-124 ${ }^{\circ} \mathrm{C}$, yield 93\%; ${ }^{1} \mathrm{H}$ NMR (400 MHz, $\left.\mathrm{CDCl}_{3}\right) \delta 8.06(\mathrm{~d}, J=7.9 \mathrm{~Hz}, 1 \mathrm{H}), 7.88(\mathrm{dd}, J$ $=13.8,8.2 \mathrm{~Hz}, 2 \mathrm{H}), 7.68-7.38(\mathrm{~m}, 6 \mathrm{H}), 6.85(\mathrm{dd}, J=8.8,2.7 \mathrm{~Hz}$, $1 \mathrm{H}), 5.51(\mathrm{~s}, 3 \mathrm{H}), 3.76(\mathrm{t}, J=5.1 \mathrm{~Hz}, 4 \mathrm{H}), 2.90-2.79(\mathrm{~m}, 2 \mathrm{H}), 2.79-$ $2.60(\mathrm{~m}, 2 \mathrm{H}), 2.16(\mathrm{~s}, 2 \mathrm{H}) ;{ }^{13} \mathrm{C} \mathrm{NMR}\left(101 \mathrm{MHz}, \mathrm{CDCl}_{3}\right) \delta 158.67$ (s), 140.15 (s), 133.81 (s), 133.54 (s), 131.65 (s), 131.49 (s), 129.26 (s), 128.75 (s), 126.91 (s), 126.60 (s), 126.02 (s), 125.25 (s), 123.65 (s), $117.12(\mathrm{~s}), 115.85(\mathrm{~s}), 114.05(\mathrm{~s}), 69.06$ (s), 61.09 (s), 51.53 (s), 36.01 (s); IR (KBr, $\mathrm{cm}^{-1}$ ) $\nu 3415.1$ (s), 3291.4 (s), 1517.8 (s), 1467.1 (s), 1016.2 (s); HRMS (ES) $m / z$ for $\mathrm{C}_{22} \mathrm{H}_{23} \mathrm{BrO}_{3} \mathrm{~S}_{2}[\mathrm{M}+\mathrm{Na}]^{+}$ cacld 501.0164, found 501.0161.

Bis(2-hydroxyethyl)-4-(naphthalen-1-ylmethoxy)-3-chlorophenyldithioacetal (S15). White solid, mp 102-104 ${ }^{\circ} \mathrm{C}$, yield 94\%; ${ }^{1} \mathrm{H}$ NMR (400 MHz, $\left.\mathrm{CDCl}_{3}\right) \delta 8.08(\mathrm{~d}, J=8.2 \mathrm{~Hz}, 1 \mathrm{H}), 7.94-7.82$ $(\mathrm{m}, 2 \mathrm{H}), 7.65(\mathrm{~d}, J=6.9 \mathrm{~Hz}, 1 \mathrm{H}), 7.61-7.43(\mathrm{~m}, 4 \mathrm{H}), 7.31(\mathrm{dd}, J=$ 8.5, $2.3 \mathrm{~Hz}, 1 \mathrm{H}), 7.05(\mathrm{~d}, J=8.5 \mathrm{~Hz}, 1 \mathrm{H}), 5.59(\mathrm{~s}, 2 \mathrm{H}), 5.04(\mathrm{~s}, 1 \mathrm{H})$, $3.76(\mathrm{q}, J=5.6 \mathrm{~Hz}, 4 \mathrm{H}), 2.85(\mathrm{dt}, J=14.0,5.8 \mathrm{~Hz}, 2 \mathrm{H}), 2.71(\mathrm{dt}, J$ $=14.0,5.9 \mathrm{~Hz}, 2 \mathrm{H}), 2.15(\mathrm{~s}, 2 \mathrm{H}) ;{ }^{13} \mathrm{C} \mathrm{NMR}\left(101 \mathrm{MHz}, \mathrm{CDCl}_{3}\right)$ $\delta 154.10$ (s), 133.74 (s), 133.63 (s), 131.55 (s), 131.23 (s), 129.71 (s), 129.05 (s), 128.74 (s), 126.98 (s), 126.49 (s), 126.16 (s), 125.96 (s), 125.31 (s), 123.69 (s), 123.44 (s), 114.08 (s), 69.68 (s), 61.47 (s), 52.25 (s), 35.59 (s); IR (KBr, cm $\left.{ }^{-1}\right) \nu 3418.8(\mathrm{~s}), 3271.4(\mathrm{~s})$, 1537.8 (s), 1467.1 (s), 1016.2 (s); HRMS (ES) $\mathrm{m} / \mathrm{z}$ for $\mathrm{C}_{22} \mathrm{H}_{23} \mathrm{ClO}_{3} \mathrm{~S}_{2}[\mathrm{M}+\mathrm{Na}]^{+}$cacld 457.0669, found 457.0663.

Bis(2-hydroxyethyl)-2-(naphthalen-1-ylmethoxy)-5-methyl-

phenyldithioacetal (S16). White solid, mp 139-140 ${ }^{\circ} \mathrm{C}$, yield $91 \%$; ${ }^{1} \mathrm{H}$ NMR (400 MHz, $\left.\mathrm{CDCl}_{3}\right) \delta$ 8.11-8.03 (m, 1H), 7.97-7.82 (m, 2H), 7.63-7.40 (m, 5H), 7.12-7.04 (m, 1H), $7.00(\mathrm{~d}, J=8.3 \mathrm{~Hz}$, $1 \mathrm{H}), 5.52(\mathrm{~s}, 2 \mathrm{H}), 5.43(\mathrm{~s}, 1 \mathrm{H}), 3.48(\mathrm{q}, J=5.9 \mathrm{~Hz}, 4 \mathrm{H}), 2.67(\mathrm{dd}, J$ $=12.8,7.0 \mathrm{~Hz}, 2 \mathrm{H}), 2.57(\mathrm{dd}, J=12.9,7.1 \mathrm{~Hz}, 2 \mathrm{H}), 2.32(\mathrm{~s}, 3 \mathrm{H})$, $1.90(\mathrm{~d}, J=6.2 \mathrm{~Hz}, 2 \mathrm{H}) ;{ }^{13} \mathrm{C} \mathrm{NMR}\left(101 \mathrm{MHz}, \mathrm{CDCl}_{3}\right) \delta 152.77(\mathrm{~s})$, 133.83 (s), 132.16 (s), 131.57 (s), 131.06 (s), 129.63 (s), 129.28 (s), 129.14 (s), 128.85 (s), 128.78 (s), 126.91 (s), 126.52 (s), 126.03 (s), 125.37 (s), 123.69 (s), 112.24 (s), 69.38 (s), 60.93 (s), 45.21 (s), 36.05 (s), 20.64 (s); IR (KBr, cm $\left.{ }^{-1}\right) \nu 3421.2$ (s), 3271.4 (s), 1547.6 (s), 1477.3 (s), 1018.2 (s); HRMS (ES) $m / z$ for $\mathrm{C}_{23} \mathrm{H}_{26} \mathrm{O}_{3} \mathrm{~S}_{2}[\mathrm{M}+$ $\mathrm{Na}]^{+}$cacld 437.1216, found 437.1219. 


\section{Biological assays}

In vivo antiviral activity. The biological activity of the title compounds against tobacco mosaic virus (TMV) and cucumber mosaic virus (CMV) respectively were evaluated using a half-leaf method according to the previously references. ${ }^{24}$ Virus purification and activity evaluation of the compounds were performed as previously reported..$^{27,28}$

In vitro antibacterial activity. The antibacterial activities against rice bacterial leaf blight of title products were evaluated via the turbid meter test according to the reported method. ${ }^{29}$ Bacterial cultivation and activity test of the title compounds were performed as previously reported. ${ }^{30}$

\section{Conflicts of interest}

The authors confirm that this article content has no conflict of interest.

\section{Acknowledgements}

The authors gratefully acknowledge financial support by the National Key Research and Development Program of China (No. 2017YFD0200506), the National Natural Science Foundation of China (No. 21807037), the National Special Fund For AgroScientific Research in the Public Interest of China (No. 201503112-8), the Provincial Major Project of Education Department in Anhui (No. KJ2018A0386), the Provincial Major Project of Excellent Youth Talent Support Program in Anhui (No. gxyqZD2018092), National innovation training program for college students (20171402085). We also sincerely thank all coworkers who have contributed to the work.

\section{Notes and references}

1 (a) J. A. Tomlinson, Ann. Appl. Biol., 1987, 110, 661; (b) A. N. Creager, K. B. Scholthof, V. Citovsky and H. B. Scholthof, Plant Cell, 1999, 11, 301; (c) M. Jacquemond, Adv. Virus Res., 2012, 84, 439.

2 (a) S. Boquel, J. Zhang, C. Goyer, M. A. Giguère, C. Clark and Y. Pelletier, Pest Manage. Sci., 2015, 71, 1106; (b) A. V. Karasev and S. M. Gray, Annu. Rev. Phytopathol., 2013, 51, 571.

3 K. B. G. Scholthof, S. Adkins, H. Czosnek, P. Palukaitis, E. Jacquot, T. Hohn, B. Hohn, K. Saunders, T. Candresse, P. Ahlquist, C. Hemenway and G. D. Foster, Mol. Plant Pathol., 2011, 12, 938.

4 H. Xiao, P. Li, D. Y. Hu and B. A. Song, Bioorg. Med. Chem. Lett., 2014, 24, 3452.

5 M. H. Chen, P. Li, D. Y. Hu and B. A. Song, Bioorg. Med. Chem. Lett., 2016, 26, 168.

6 J. Wu and B. A. Song, Sci. Sin.: Chim., 2016, 46, 1165.

7 B. A. Song, S. Yang, L. H. Jin and P. S. Bhadury, Environment friendly antiviral agents for plants, Springer, Berlin, 2009.

8 Z. Z. Wang, D. D. Xie, X. H. Gan, D. Y. Hu and B. A. Song, Bioorg. Med. Chem. Lett., 2017, 27, 4096.
9 J. D. Jones and J. L. Dangl, Nature, 2006, 444, 323.

10 F. P. Silverman, P. D. Petracek, Z. Ju, C. M. Fledderman, D. F. Heiman and P. Warrior, J. Agric. Food Chem., 2005, 53, 9764.

11 H. Kauss, K. Krause and W. Jeblick, Biochem. Biophys. Res. Commun., 1992, 189, 304.

12 Z. J. Fan, Z. G. Shi, H. K. Zhang, X. F. Liu, L. L. Bao, L. Ma, X. Zuo, Q. X. Zheng and N. Mi, J. Agric. Food Chem., 2009, $57,4279$.

13 F. P. Silverman, P. D. Petracek, Z. Ju, C. M. Fledderman, D. F. Heiman and P. Warrior, J. Agric. Food Chem., 2005, 53, 9769.

14 F. P. Silverman, P. D. Petracek, D. F. Heiman, C. M. Fledderman and P. Warrior, J. Agric. Food Chem., 2005, 53, 9775.

15 M. Lhassani, O. Chavignon, J. M. Chezal, J. C. Teulade, J. P. Chapat, R. Snoeck, G. Andrei, J. Balzarini, E. D. De Clercq and A. Gueiffier, Eur. J. Med. Chem., 1999, 34, 271.

16 L. Sakthikumar, J. Kavitha and R. Mahalakshmi, Der Pharma Chem., 2014, 6, 294.

17 H. Koga, Y. Tsuji, K. Inoue, K. Kanai, T. Majima, T. Kasai, K. Uchida and H. Yamaguchi, J. Infect. Chemother., 2006, 12, 163.

18 H. Koga, Y. Nanjoh, K. Makimura and R. Tsuboi, Med. Mycol., 2009, 47, 640.

19 S. Pandey, S. Suryawanshi, S. Gupta and V. Srivastava, Eur. J. Med. Chem., 2005, 40, 751.

20 J. Zhang, L. Zhao, C. Zhu, Z. X. Wu, G. P. Zhang, X. H. Gan, D. Y. Liu, J. K. Pan, D. Y. Hu and B. A. Song, J. Agric. Food Chem., 2017, 65, 4582.

21 J. Chen, J. Shi, L. Yu, D. Y. Liu, X. H. Gan, B. A. Song and D. Y. Hu, J. Agric. Food Chem., 2018, 66, 5335.

$22 \mathrm{H}$. C. Zhang and X. L. Li, Instruction manual for plant growth regulators, China Agriculture Press, 2011.

23 K. K. Pasunooti, H. Chai, C. N. Jensen, B. K. Gorityala, S. Wang and X. W. Liu, Tetrahedron Lett., 2011, 52, 80.

24 Z. W. Wang, A. Feng, M. Cui, Y. Liu, L. Wang and Q. M. Wang, PLoS One, 2012, 7, e52933.

25 S. G. Bhansali, et al., Int. J. PharmTech Res., 2013, 5, 1224.

26 G. V. Gooding Jr and T. T. Hebert, Phytopathology, 1967, 57, 1285.

27 H. Scott, Virology, 1963, 20, 103.

28 D. G. Zhou, D. D. Xie, F. C. He, B. A. Song and D. Y. Hu, Bioorg. Med. Chem. Lett., 2018, 28, 2091.

29 Z. X. Wu, J. Zhang, J. X. Chen, J. K. Pan, L. Zhao, D. Y. Liu, A. W. Zhang, J. Chen, D. Y. Hu and B. A. Song, Pest Manage. Sci., 2017, 73, 2079.

30 (a) Z. H. Ma, M. G. Zhou and Z. Y. Ye, Acta Phytopathol. Sin., 1997, 27, 237; (b) G. B. Shen and M. G. Zhou, Plant Prot., 2002, 28, 9.

31 P. Li, D. Y. Hu, D. D. Xie, J. X. Chen, L. H. Jin and B. A. Song, J. Agric. Food Chem., 2018, 66, 3093. 